\title{
Implementation and evaluation of coherent synthetic aperture radar processing for level measurements of bulk goods with an FMCW-system
}

\author{
M. $\operatorname{Vogt}^{1}$, M. Gerding ${ }^{2}$, and T. Musch ${ }^{2}$ \\ ${ }^{1}$ Forschungsgruppe Hochfrequenztechnik, Ruhr-Universität Bochum, 44780 Bochum, Germany \\ ${ }^{2}$ Lehrstuhl für Elektronische Schaltungstechnik, Ruhr-Universität Bochum, 44780 Bochum, Germany
}

\begin{abstract}
In industrial process measurement instrumentation, radar systems are well established for the measurement of filling levels of liquids in tanks. Level measurements of bulk goods in silos, on the other hand, are more challenging because the material is heaped up and its surface has typically a relatively complex shape. In this paper, the application of synthetic aperture radar (SAR) reconstruction with a frequency modulated continuous wave (FMCW) radar system for level measurements of bulk goods is evaluated. In the proposed monostatic setup, echo signals are acquired at discrete antenna positions on top of the silo. Spatially resolved information about the surface contour of a bulk good heap is reconstructed by coherent 'delay and sum' processing. The concept has been experimentally evaluated with a 24 to $26 \mathrm{GHz}$ FMCW radar system mounted on a linear stepping motor positioning unit. Measurements on a thin metal wire at different range and on a curved test-object with a diffusely scattering surface have been performed to analyze the system's point spread function (PSF) and performance. Constant range and azimuth resolutions $(-6 \mathrm{~dB})$ of $15 \mathrm{~cm}$ and $8 \mathrm{~cm}$, respectively, have been obtained up to a range of $6 \mathrm{~m}$, and results of further evaluations show that the proposed concept allows more accurate and reliable level reconstructions of surface profiles compared to the conventional approach with measurements at a single antenna position.
\end{abstract}

\section{Introduction}

The conventional approach in radar and time-domain reflectometry (TDR) level measurement is to perform echo measurements along a fixed radiation beam with an antenna at a fixed position on top of a tank or silo (Reindl et al., 2001;

Correspondence to: $\mathrm{M}$. Vogt

(michael.vogt@ rub.de)
Gerding et al., 2003, 2006; Rabe et al., 2009). In the case of liquids, this technique usually delivers adequate and sufficiently good results, because the surface of the liquid is planar and the surface level is the only relevant parameter. Level measurements on bulk goods are more challenging because the material is heaped up and its surface profile is aimed with the measurement.

Figure 1 shows the scenario given with radar level measurements of liquids in tanks in comparison to bulk goods in silos. In Fig. 1a, microwaves are emitted from an antenna at a fixed position on top of the tank and are reflected at the planar boundary between the air and the liquid. From the time of flight (TOF) of propagating waves, the distance between the antenna and the boundary is measured, and the filling level $H$ of the liquid can be calculated by taking the overall height of the tank into account. Different from ultrasound based systems, radar level measurements are largely independent from the composition of the air, fog, temperature, pressure, etc., and high precision measurements can be performed. A single-point measurement delivers sufficient information in the case of level measurements of liquids, because only a single parameter, the filling level $H$, is relevant.

Level measurements on bulk goods, on the other hand, are much more challenging because the material is heaped up and its surface typically builds a complicated shape, as is shown in Fig. 1b. Microwaves are diffusely scattered at the boundary between the air and the bulk good, and, in general, penetrate into the bulk good and are diffusely scattered inside the heap. Typically, the amplitude of echo signals from bulk goods is much smaller compared to echoes from strong reflecting surfaces like boundaries between air and water. With respect to the measurement problem, which is to assess the bulk good volume inside the silo, a single-point measurement is insufficiently in many cases because of the more complicated geometry, see Fig. 1b. 
a)

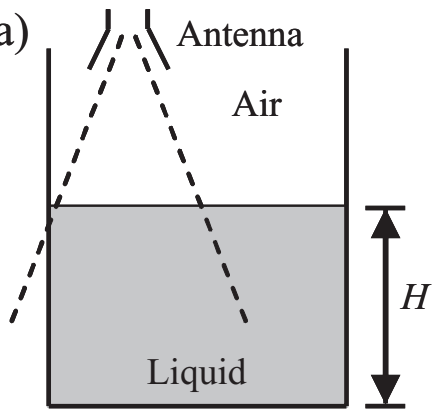

b)

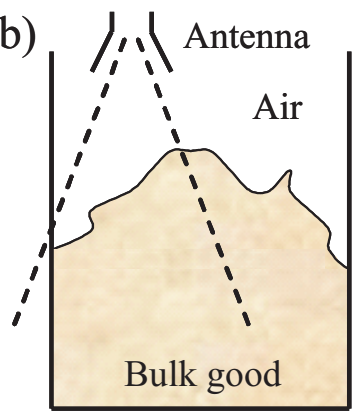

Fig. 1. Radar level measurement: (a) Liquid in tank, (b) Bulk good in silo.

In this paper, the utilization of synthetic aperture radar (SAR) processing for the imaging and assessment of surface profiles of bulk goods with a frequency modulated continuous wave (FMCW) radar system is evaluated. In the SAR approach, echo signals are acquired along a relatively long azimuth scanning path with a single antenna, and signals are superimposed after correction for the TOFs the waves need to travel on the round-trip between the antenna and each individual image point to be reconstructed. Coherent 'delay and sum' processing in the time-domain is applied for highresolution reconstruction of information about reflection and scattering properties of objects. Calculations and measurements have been performed for the specific test-setup presented below.

\section{Scanning radar system}

Echo measurements at multiple antenna positions on top of a silo are proposed as a concept to cope with the aforementioned problems. SAR processing is applied to reconstruct backscattering and reflection images of the bulk goods from acquired echo signal data (Brenner and Roessing, 2008; Meta et al., 2007; Charvat et al., 2006; Ermert and Karg, 1979).

\subsection{System setup}

In the monostatic radar system configuration in Fig. 2, microwaves are emitted from a horn antenna, and backscattered and reflected waves are received by the same antenna.

Echo signals at discrete antenna positions $x_{a}$ are sampled, digitized and stored for SAR processing and the reconstruction of two-dimensional (2-D) images of backscattering and reflecting structures in the $2-\mathrm{D} x / z$ image plane.

\subsection{System's point spread function (PSF)}

Below, time-domain receive signals over TOF $t$ at aperture positions $x_{\mathrm{a}}$ for a single point-object, i.e. a point-like radar target, which is small against the wavelength, at a position

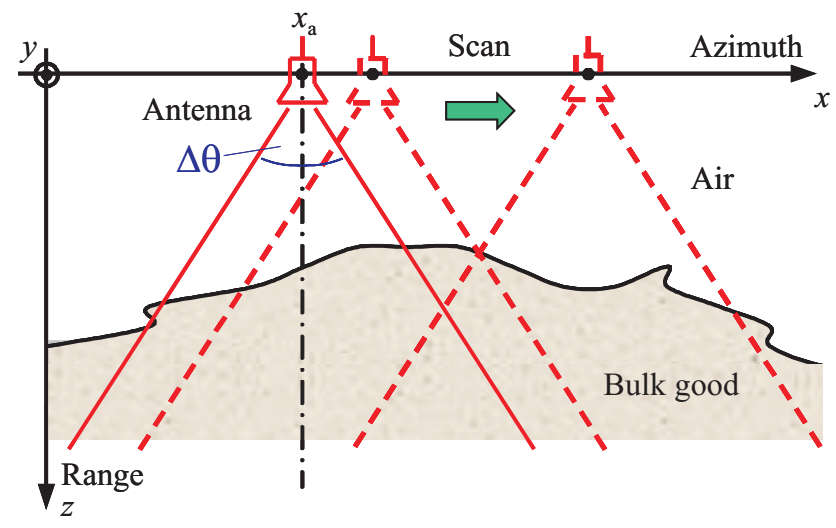

Fig. 2. Scanning radar system in monostatic system configuration.

$\left(x^{\prime}, z^{\prime}\right)$ are denoted as the system's point spread function (PSF) $h\left(t, x_{\mathrm{a}}, x^{\prime}, z^{\prime}\right)$ (Vogt et al., 2010). For point-objects in the far field of the antenna, the PSF can be described as follows, see Fig. 2:

$$
\begin{aligned}
& h\left(t, x_{\mathrm{a}}, x^{\prime}, z^{\prime}\right)=s\left(t-2 / c \cdot r\left(x_{\mathrm{a}}, x^{\prime}, z^{\prime}\right)\right) \\
& \text { with }: r\left(x_{\mathrm{a}}, x^{\prime}, z^{\prime}\right)=\sqrt{z^{\prime 2}+\left(x_{\mathrm{a}}-x^{\prime 2}\right)}
\end{aligned}
$$

In Eq. (1), $r\left(x_{\mathrm{a}}, x^{\prime}, z^{\prime}\right)$ is the radial distance between the antenna and the point-object, $c$ the speed of light, and the pulsed signal $s(t)$ is the impulse response of the band-pass radar system. Both, the radial distance $r\left(x_{\mathrm{a}}, x^{\prime}, z^{\prime}\right)$ as well as the PSF $h\left(t, x_{\mathrm{a}}, x^{\prime}, z^{\prime}\right)$, are invariant against translations of the point-object along the azimuth direction $x$ (Vogt et al., 2010):

$$
\begin{aligned}
r\left(x_{\mathrm{a}}, x^{\prime}, z^{\prime}\right) & =r^{\prime}\left(x_{\mathrm{a}}-x^{\prime}, z^{\prime}\right) \\
h\left(t, x_{\mathrm{a}}, x^{\prime}, z^{\prime}\right) & =h^{\prime}\left(t, x_{\mathrm{a}}-x^{\prime}, z^{\prime}\right)
\end{aligned}
$$

Echo signals from any backscattering or reflecting radar target can be described based on the composition of objects by discrete point-objects and the corresponding superposition of PSFs, as long as multiple reflections are negligible and the radar system is linear (Opretzka et al., 2010).

\section{SAR reconstruction}

In the implemented radar system, SAR processing is performed by 'delay and sum' operation in the time-domain. 2-D backscattering and reflection images $b(x, z)$ are reconstructed from the acquired receive signals $s_{\mathrm{R}}\left(t, x_{\mathrm{a}}\right)$ at aperture positions $x_{\mathrm{a}}$ as follows (Vogt et al., 2010):

$$
\begin{aligned}
b(x, z)= & \mid \sum_{n=n_{1}}^{n_{2}} w\left(n-n_{1}\right) \\
& \left.\cdot s_{\mathrm{R}+}\left(t+\tau\left(x_{\mathrm{a}, n}, x, z\right), x_{\mathrm{a}, n}\right)\right|_{t=2 / c \cdot z^{\prime}}
\end{aligned}
$$

with: $\quad \tau\left(x_{\mathrm{a}, n}, x, z, x_{\mathrm{a}, n}\right)=2 / c \cdot\left(r\left(x_{\mathrm{a}, n}, x, z\right)-z^{\prime}\right)$ 
In Eq. (3), $s_{\mathrm{R}+}(t)$ is the analytical receive signal obtained from the receive signal $s_{\mathrm{R}}(t)$ by means of the Hilbert Transform. SAR reconstruction is performed by delaying the acquired receive signals at antenna positions $x_{\mathrm{a}, n}$ by $\tau\left(x_{\mathrm{a}, n}, x, z\right)$ and coherent summation of delayed signals after apodization with a weighting function $w(n)$. The backscattering and reflection image $b(x, z)$ is calculated as the magnitude of the analytical focused receive signal obtained after delay and sum operation. The summation in Eq. (3) is extended over receive signals from within the antenna's main lobe (aperture angle $\Delta \Theta$, see Fig. 2), which corresponds to a summation along a synthetic aperture of length $L_{\mathrm{SAR}}(z) \approx z^{\prime} \cdot \Delta \Theta$ along the azimuth direction $x$.

\section{Evaluation of proposed imaging concept}

The proposed imaging concept has been evaluated by measurements with an FMCW radar system, which was mounted on a linear stepping motor positioning unit.

\subsection{FMCW radar system}

In Fig. 3a, the block diagram of the utilized FMCW radar system $(25 \mathrm{GHz}$ center frequency, $2 \mathrm{GHz}$ bandwidth, $8 \mathrm{~ms}$ sweep time, $1 \mathrm{MHz}$ intermediate signal sampling frequency) is shown (Musch, 2003).

With this system, the transfer function $H(j \omega)$ of the radar path is assessed by the intermediate frequency (IF) echo signal $s_{\mathrm{IF}}(t)$. The latter is obtained by mixing the frequency modulated transmit signal $s_{\mathrm{T}}(t)$ with linearly swept instantaneous frequency $\omega(t)$ with the echo signal $s_{\mathrm{E}}(t)$ :

$$
\begin{aligned}
s_{\mathrm{T}}(t) & =a_{0} \cdot \cos \left(\varphi_{\mathrm{T}}(t)\right), \omega(t)=2 \pi \cdot\left(f_{1}+B / T \cdot t\right) \\
\varphi_{\mathrm{T}}(t) & =2 \pi \cdot\left(f_{1} \cdot t+b /(2 \cdot T) \cdot t^{2}\right)+\varphi_{0} \\
s_{\mathrm{E}}(t) & \approx a_{0} \cdot|H(j \omega(t))| \cdot \cos \left(\varphi_{\mathrm{T}}(t)+\arg (H(j \omega(t)))\right) \\
s_{\mathrm{IF}}(t) & \approx k \cdot a_{0} \cdot|H(j \omega(t))| \cdot \cos (\arg (H(j \omega(t)))) \\
s_{\mathrm{IF}}(t) & \sim \operatorname{Re}\{H(j \omega(t))\}
\end{aligned}
$$

It can be seen in Fig. $3 \mathrm{~b}$ that the echo signal $s_{\mathrm{E}}(t)$ also shows a linearly swept instantaneous frequency $\omega_{\mathrm{E}}(t)$, but with a constant delay $\tau$ for a single radar target. The receive signal $s_{\mathrm{R}}(t)$ containing the impulse response $h(t)$ of the radar path is obtained from the Fourier Transform of $s_{\mathrm{IF}}(t)$ as follows, see Eq. (4):

$$
\begin{aligned}
& S_{\mathrm{R}}(\omega)=s_{\mathrm{IF}}(t)_{t \rightarrow \omega(t)}=\operatorname{Re}\{H(j \omega(t))\} \\
& S_{\mathrm{R}}(\omega)=\frac{1}{2} \cdot\left(H(j \omega)+H^{*}(j \omega)\right) \\
& s_{\mathrm{R}}(t)=\frac{1}{2} \cdot\left(h(t)+h^{*}(-t)\right)
\end{aligned}
$$

\subsection{Measurement setup}

The FMCW radar system was equipped with a conical horn antenna with $75 \mathrm{~mm}$ aperture diameter and mounted on a a)

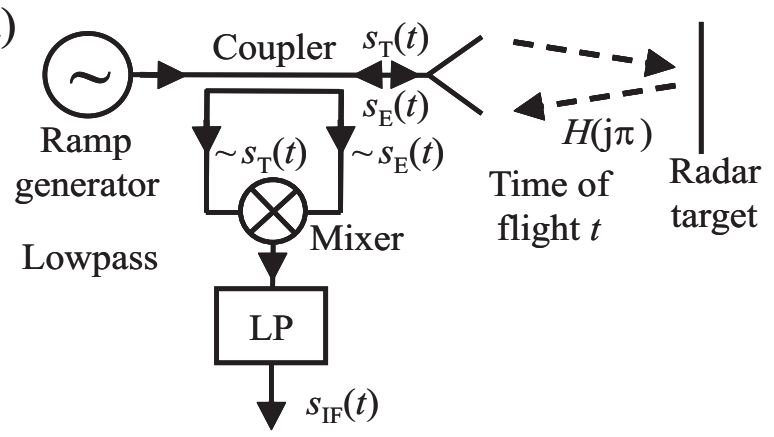

b)

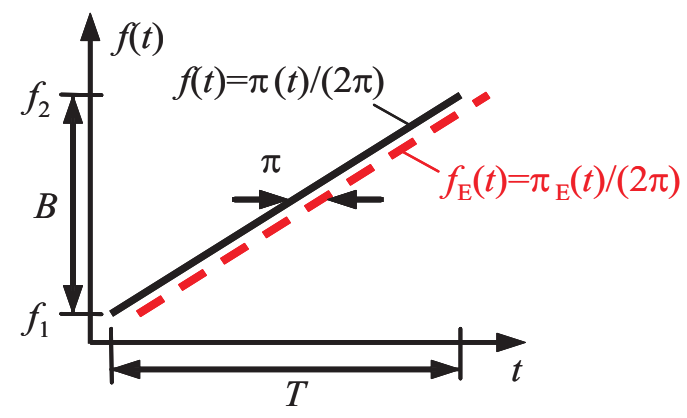

Fig. 3. FMCW radar system: (a) Block diagram, (b) Instantaneous frequencies $f(t)$ and $f_{\mathrm{E}}(t)$ of transmit and echo signal, respectively, for a single radar target.

linear stepping motor positioning unit. Echo measurements have been performed at 256 equidistant antenna positions with a distance of $6.6 \mathrm{~mm}$ from each other $(55 \%$ of wavelength at $25 \mathrm{GHz}$ center frequency), covering a scanning path with a length of $1.7 \mathrm{~m}$. A thin metal wire ( $3 \mathrm{~mm}$ diameter) has been imaged at different range in order to assess the system's PSF. Furthermore, a test-object with a curved profile along the azimuth scanning direction and a diffusely scattering surface has been utilized to evaluate the proposed concept with respect to applications in bulk good level measurements.

\section{Measurement results}

In Fig. 4a, a schematic of the laboratory environment for the PSF measurements with concrete walls at the sides of the laboratory room are shown.

The metal wire was positioned along the elevational direction $y$ at a chosen distance $z$ in range direction mounted on a tripod with an arm, and scanned along the azimuth direction $x$. Under these conditions, the metal wire is a point-like object in the $x / z$ azimuth/range image plane. Consequently, the system's PSF can be analyzed by echo measurements from the wire positioned at different range $z$. In the unfocused 2-D backscattering and reflection image $b(x, z)$ in Fig. $4 \mathrm{~b}$, the envelope of the receive signals without delay and sum focusing is shown over a dynamic range of $30 \mathrm{~dB}$. Echoes from the metal wire at a range of $5.5 \mathrm{~m}$ are spread along the azimuth 


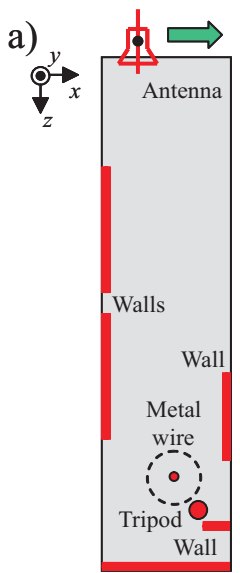

b)

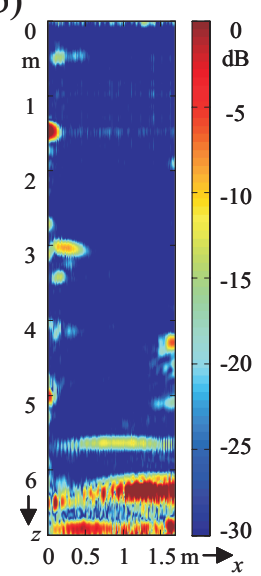

c)

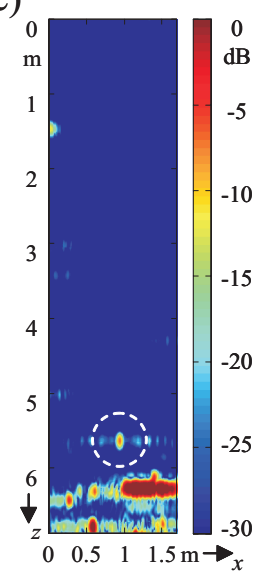

Fig. 4. Point spread function (PSF) measurement: (a) Schematic of measurement environment (laboratory), (b) Unfocused radar image, (c) Focused radar image.

coordinate $x$ due to the diverging beam pattern of the antenna in the far field. Furthermore, echoes from the corners of the side wall are visible, as well as strong reflections from the back wall. In Fig. 4c, the focused backscattering and reflection image $b(x, z)$ of the same scenario obtained by SAR processing is shown. Now, the result is a point-like, focused image of the metal wire in the $x / z$ azimuth/range image plane, and echoes from the corners of the side walls are suppressed.

The $-6 \mathrm{~dB}$-widths of the echo from the wire along the azimuth and range coordinates $x$ and $z$, respectively, have been assessed in cross-sectional images at different range $z$ of the wire in order to analyze the system's range and azimuth resolutions $\delta_{z}$ and $\delta_{x}$, respectively (Opretzka et al., 2010). Results are shown in Fig. 5.

Almost constant range resolutions $(-6 \mathrm{~dB})$ of $15 \mathrm{~cm}$ are obtained in the unfocused and focused images up to a range of $6 \mathrm{~m}$ (solid line and dotted/dashed line). This is in good agreement with the expectation that the range resolution only depends on the pulse-width of the system's impulse response, which is inversely proportional to the constant bandwidth of the system of $2 \mathrm{GHz}$. The azimuth resolution in unfocused images (dashed line), on the other hand, is proportional to depth, because of the diverging beam pattern (constant aperture angle $\Delta \Theta$ ) in the far field of the antenna. As indicated by the example in Fig. $4 c$, a much smaller and almost constant azimuth resolution $(-6 \mathrm{~dB})$ of $8 \mathrm{~cm}$ is obtained in focused images up to a range of $6 \mathrm{~m}$ with the implemented system (dotted line).

The setup shown in Fig. 6a has been used to evaluate the proposed concept in the context of level measurements on bulk goods. A test-object with a curved profile along the azimuth scanning direction and a diffusely scattering surface has been created by gluing a crunched aluminum foil on a pa-

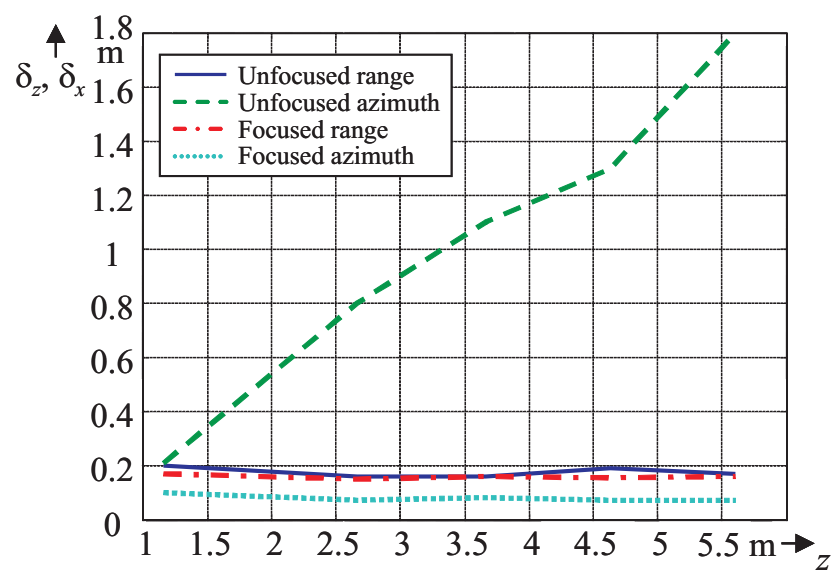

Fig. 5. Results of point spread function (PSF) measurements: Range resolution $\delta_{z}$ and azimuth resolution $\delta_{x}$ (FWHM) in unfocused and focued radar images over range $z$.

perboard. This structure has been bended along the azimuth coordinate $x$, as is shown in Fig. 6a, and was positioned at a mean range of about $5.3 \mathrm{~m}$.

The unfocused 2-D backscattering and reflection image $b(x, z)$ in Fig. $6 \mathrm{~b}$ again shows the envelope of receive signals without delay and sum focusing. It can be seen that the image contains information about the curved surface profile of the test-object. As a result of the superposition of diffusely backscattered coherent waves, 'speckle' patterns appear in the image. Furthermore, because of the relatively bad azimuth resolution, echoes from the surface of the test object along the azimuth coordinate, i.e. echoes from different depths, are superimposed in the unfocused image. Consequently, a precise detection of the object's surface is difficult. In the focused image in Fig. 6c of the same scenario, the speckle and the surface profile are less spread over range and azimuth directions compared to the unfocused image in Fig. 6b. Consequently, the surface of the object can be more easily and more accurately detected from the focused image.

\section{Summary and conclusions}

In this paper, the application of SAR processing has been proposed and evaluated to cope with the problem of radar level measurements of bulk goods in silos, which is challenging compared to level measurements of liquids in tanks. Echo measurements with a single antenna in a monostatic configuration along a 1-D scanning path have been discussed as a concept for the reconstruction of 2-D backscattering and reflection images of bulk goods. SAR processing in the time-domain by 'delay and sum' operation has been suggested for this purpose. The concept has been evaluated by measurements with a 24 to $26 \mathrm{GHz}$ frequency range FMCW radar system, which was equipped with a conical 
a)

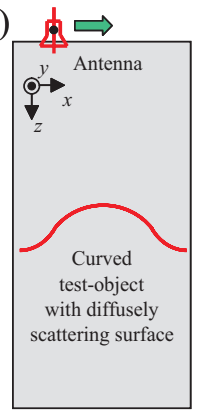

b)

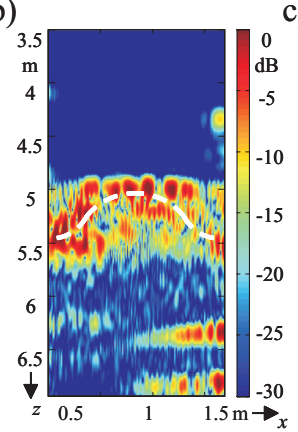

c)

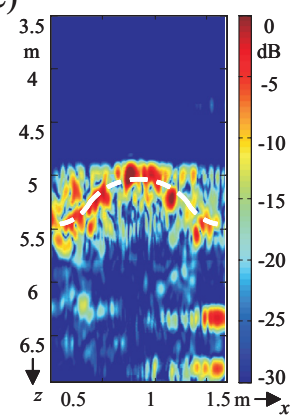

Fig. 6. Measurement on curved test-object: (a) Schmeatic of measurement environment (laboratory), (b) Unfocused radar image, (c) Focused radar image.

horn antenna with $75 \mathrm{~mm}$ aperture diameter. In the implemented system, the antenna is scanned along the azimuth coordinate by means of a linear stepping motor positioning unit, and 2-D backscattering and reflection images inside the azimuth/range image plane are reconstructed. Based on echo measurements on a metal wire, the system's PSF has been evaluated, showing that constant range and azimuth resolutions $(-6 \mathrm{~dB})$ of $15 \mathrm{~cm}$ and $8 \mathrm{~cm}$, respectively, are achieved up to a range of $6 \mathrm{~m}$. Results of measurements on a curved test-object with diffusely scattering surface show that the implemented system along with the proposed SAR concept allows to accurately assess curved profiles from reconstructed backscatter and reflection images. The goal of our future work is to extend the concept towards a 2-D scanning and 3 -D SAR reconstruction and to further evaluate the proposed concept.

\section{References}

Brenner, A. R. and Roessing, L.: Radar imaging of urban areas by means of very high-resolution SAR and interferometric SAR, IEEE Trans. Geosc. Remote Sensing, 46(10), 2971-2982, 2008.

Charvat, G. L. and Kempel, L. C.: Synthetic aperture radar imaging using a unique approach to frequency-modulated continuouswave radar design, IEEE Ant. Prop. Magazine, 48(1), 171-177, 2006.

Ermert, H. and Karg, R.: Multifrequency acoustical holography, IEEE Trans. Sonics, Ultrasonics, SU-26, 4, 279-286, 1979.

Gerding, M., Musch, T., and Schiek, B.: Precision level measurement based on time-domain reflectometry, Adv. Radio Sci., 1, 27-31, 2003, http://www.adv-radio-sci.net/1/27/2003/.

Gerding, M., Musch, T., and Schiek, B.: A novel approach for a high-precision multitarget-level measurement system based on time-domain reflectometry, IEEE Trans. Instr. Meas., 54(6), 2768-2773, 2006.

Meta, A., Hoogeboom, P., and Ligthart, L. P.: Signal processing for FMCW SAR, IEEE Trans. Geosc. Remote Sensing, 45(11), 3519-3532, 2007.

Musch, T.: A high precision 24-GHz FMCW radar based on a fractional-N ramp-PLL, IEEE Trans. Instr. Meas., 52(2), 324327, 2003.

Opretzka, J., Vogt, M., and Ermert, H.: A model-based synthetic aperture imaging reconstruction technique for high-frequency ultrasound, IEEE 2009 Ultrason. Symp. Proc., 377-380, 2010.

Rabe, H., Denicke, E., Armbrecht, G., Musch, T., and Rolfes, I.: Considerations on radar localization in multi-target environments, Adv. Radio Sci., 7, 5-10, 2009, http://www.adv-radio-sci.net/7/5/2009/.

Reindl, L., Ruppel, C. W., Berek, S., Knauer, U., Vossiek, M., Heide, P., and Oréans, L.: Design, fabrication, and application of precise SAW delay lines used in a FMCW radar system, IEEE Trans. Microw. Theory Tech., 49(4), 787-794, 2001.

Vogt, M., Opretzka, J., and Ermert, H.: Synthetic aperture focusing technique for high-resolution imaging of surface structures with high-frequency ultrasound, IEEE 2009 Ultrason. Symp. Proc., 1514-1517, 2010. 